\title{
SUPPORTING THE VENTILATOR CHALLENGE DURING THE COVID-19 PANDEMIC WITH DISCRETE EVENT SIMULATION MODELLING
}

\author{
Matthew Windeler \\ Michael Higgins \\ Giles Thomas \\ Ford Motor Company \\ Dunton Technical Centre, Essex, UK. \\ mhiggi37@ford.com \\ mwindele@ford.com \\ gthom242@ford.com
}

\begin{abstract}
A Discrete Event Simulation (DES) model was developed by Powertrain Operations Manufacturing Engineering (PTME) in support of the Ventilator Challenge production line at a Ford Motor Company facility. The facility needed to be installed and commissioned both quickly and efficiently, with DES being one of the key tools utilised to achieve this. A flexible, dynamic input model with Excel-based data input and results dashboard enabled rapid changes to the model, enabling a clear understanding of the impact of evolving factors such as operator efficiency, labour requirements and part quality throughout the project's duration. By adopting different simulation concepts the model was applied across the entire product lifecycle, from conceptualisation to decommissioning. Through the development and implementation of this model, the characteristics of the production facility were better understood, allowing targeted engineering actions to improve productivity. This project also highlighted some key learnings for applying DES within rapidly changing manufacturing environments.
\end{abstract}

Keywords: Discrete Event Simulation, Manufacturing, Ventilator, COVID-19

\section{INTRODUCTION}

In March 2020, the MRC Centre for Global Infectious Disease Analysis at Imperial College London released a report describing how their epidemiological modelling predicted that unmitigated, the demand for critical care (ICU) hospital beds (with mechanical ventilation capability) in the UK due to COVID-19 would be in excess of 30 times the current NHS capacity (Ferguson et al., 2020). It was apparent that in addition to 'flattening the curve' it was equally necessary to 'raise the line', and with a global shortage of medical ventilators - and existing manufacturers' production capabilities insufficient to meet even a fraction of demand - the Government requested assistance from businesses to support in the production and supply of ventilators to the NHS (Department for Business, Energy \& Industrial Strategy, 2020).

In response, VentilatorChallengeUK - a consortium of leading UK automotive, aerospace, and medical engineering companies - was formed, committed to delivering on formal orders from the Government for over 10,000 medical ventilators (Ventilator Challenge UK, media information notice: https://ventilatorchallengeuk.com, accessed February 2021). Ford Motor Company was part of this consortium, and many employees across Ford of Europe - including individuals and teams from Ford Powertrain Manufacturing Engineering (PTME) - were redeployed from key company projects to collaborate full-time on this initiative, throughout its duration.

While every other group responding to the Government's request attempted to develop entirely new prototype ventilators, VentilatorChallengeUK focussed instead on proven existing designs, and how production could be upscaled. Elements of the Penlon AV-S anaesthesia ventilator were redesigned to 
allow more rapid build, and with a viable process established, multiple new facilities were set up to manufacture parts, and assemble the new ESO 2 'Emergency Ventilator' in larger numbers (Penlon, VentilatorChallengeUK and the ESO 2 Emergency Ventilator: https://www.penlon.com/Blog/May2020/Penlon,-VentilatorChallengeUK-and-the-ESO-2-Emerge, accessed February 2021). Ford undertook the responsibility for the assembly and testing of these ESO 2 ventilator units (and front screens) and repurposed a facility at the Ford Dagenham plant in order to support this. As the consortium's key message stated, "every ventilator built has the potential to save a life", and it was therefore of paramount importance that the facility was installed quickly, and was capable of meeting the required capacity as soon as possible. Every available resource was put at the team's disposal in order to achieve that goal.

Proving out proposed manufacturing facilities using Discrete Event Simulation (DES) models has been a fundamental requirement for all Ford PTME projects over the last decade, and simulation modelling of such facilities has been an approach used within PTME as early as the 1970s (Ladbrook and Januszczak, 2001). By utilising the modelling skills developed for automotive manufacture and applying them to the production of ventilators, the intention was to establish the capability of the planned facility per the design, and continue to optimise it during the commissioning phase. By leveraging real data collected from the facility and feeding it back into the DES model, the production team could make informed decisions through an iterative, data-driven process.

The aim of this paper is to demonstrate how DES was applied at different phases of the project, working within a compressed time frame and with non-ideal systems, to support the effective delivery of the EOS 2 ventilator facility.

\section{METHODOLOGY}

The preferred process simulation software application of the PTME team is Lanner's Witness Horizon (Lanner, Witness Simulation Software: https://www.lanner.com/en-us/technology/witness-simulationsoftware.html, accessed February 2021) modelling studio, and it is within this technology that the team had the required expertise. As such, it was the platform used for all simulation modelling conducted during this project.

The ESO 2 ventilator facility could be considered as two distinct areas, 'Assembly', and 'Test', with these areas then split further into smaller zones as summarised in Figure 1. Each zone was characterised by a sequence of process steps assigned to manual work stations. Each unit, or part thereof, was required to pass through each step of the process. An initial model was constructed, based upon the part flow and process defined by the engineering team, with provision for removal, regression, repair and reinsertion of parts at various stages of the process, as required.

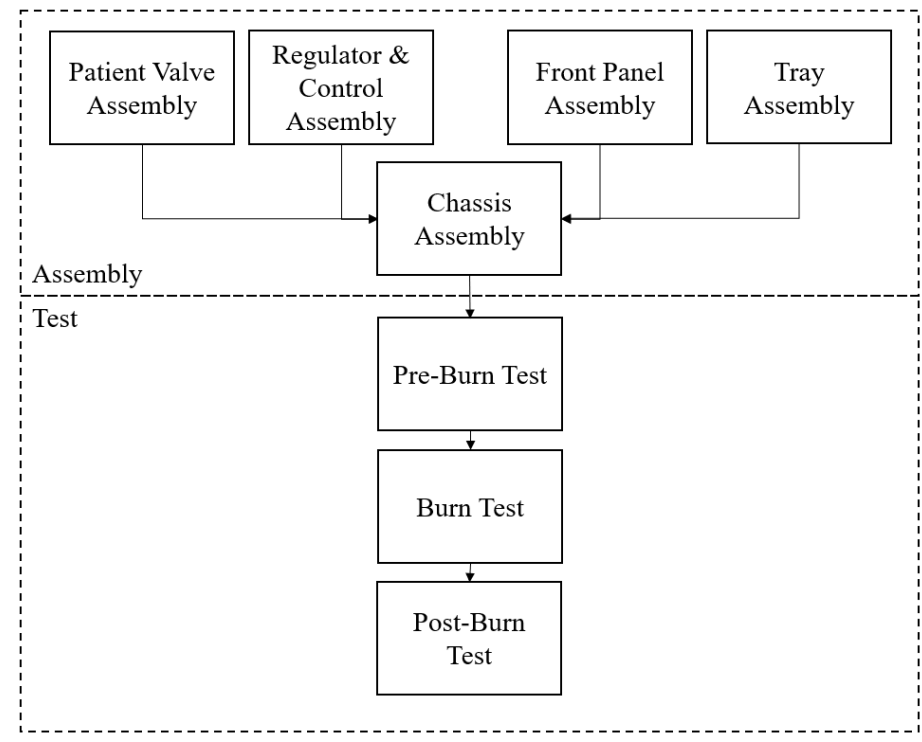

Figure 1. Simple overview of the main ESO 2 ventilator facility zones 


\section{IMPLEMENTATION}

The first challenge faced by the simulation team was the lack of a stable system description. While the wider engineering team were working with the ventilator OEM (Penlon), understanding and refining the manufacturing process, the system design was in a constant state of flux. Recognising this rapid process evolution - additional stations, workload rebalancing, modified part flow, buffer relocation would be an inherent feature of this project, the model was built to allow maximum flexibility and adaption of these changes as they were made. By creating a model with many variables and arrays that could be initialised at model start time $(\mathrm{T}=0)$ directly from an Excel workbook 'Front End', itself with links to numerous data sources, changes to the model could be made very quickly and easily, in addition to opening up further potential for model experimentation, data analysis, and optimisation.

As well as layout and flow process, there were other key input data to consider for the model too. Each station had a design cycle time (the time the station was engineered to run at). However, with the objective of the model being to better understand the inherent potential variability in the system, entering a single value for cycle time would not be adequate. For conventional models, Ford's established practice is to utilise cycle time distributions based upon recorded real-world data, either from monitoring systems on the line being modelled or a suitable surrogate operation (in the event of an entirely novel system) (Higgins, 2013). For this project, however, neither of these options were possible from the outset, so a suitable alternative was to be established. By introducing an Erlang probability density function with appropriate selection of k-value, a portion of each station cycle time was distributed relative to the design cycle time to introduce random variation about the mean, skewed toward likely overycycling - an Erlang distribution is commonly used to represent the time to complete a complex task (Robinson, 2007). This can be seen in Figure 2. The mean (CT), ratio (CTa/CTb), and $\mathrm{k}$-value inputs for the Erlang were controllable by independent model variables, enabling sensitivity analysis to be conducted on the degree of overcycles observed. There were also stations that could generate rejected parts. These were initially based on estimates from the test specialist and were presented as a percentage likelihood of a part passing through a station without issue. By using dynamic model variables to control this too, sensitivity analysis could be performed on rejection rates throughout the model run.

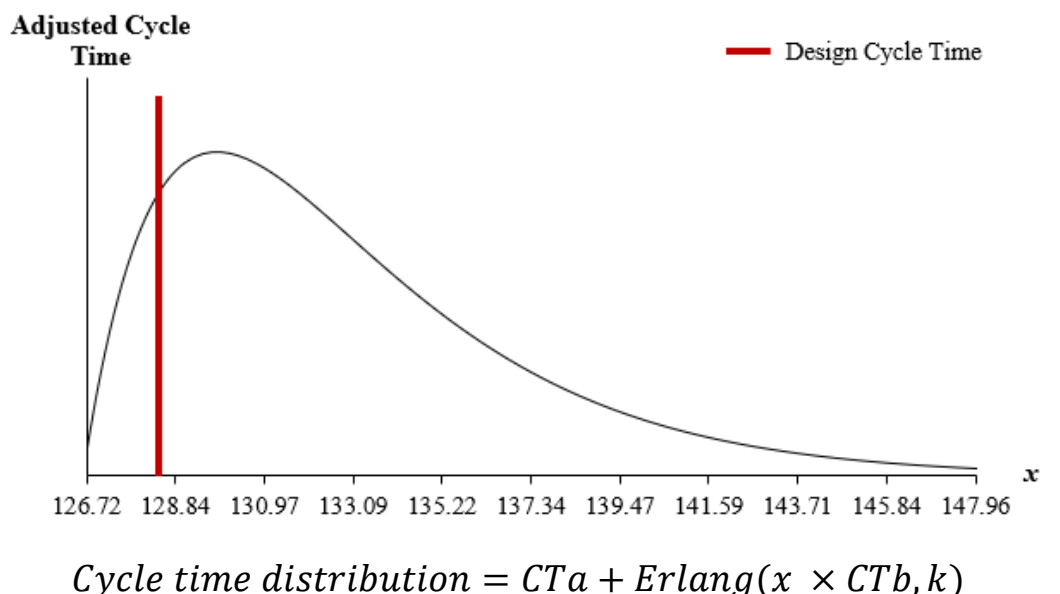

Figure 2. Erlang distribution modification of design cycle time $(\mathrm{k}=2)$

As previously mentioned, the model flexibility was a key consideration. While initially the model was built on assumptions for cycle time and reject rate, there were concurrent workstreams assigned to implementing data collection methods within the physical facility. As production commenced and progressed, a dataset would begin to grow that could then be fed back into the model to hone model accuracy and increase confidence in reported results and recommendations.

Despite the high number of initial assumptions made and the continually evolving process during the initial model build, both the model approach and inputs were discussed and agreed with key 
stakeholders as early as possible to ensure viable model progression and buy-in to the results. Whenever necessary, further detailed reviews were carried out when significant changes occurred to ensure the model remained an accurate-as-possible representation of the facility. The resulting simulation model was utilised throughout the lifecycle of the project to help characterise and optimise the line.

\subsection{Planning Phase}

In the planning phase, while the process was being developed and provisions were being made for repair areas and labour, the simulation was utilised to validate decisions and identify areas of the line that could be problematic, or might prove to be problematic should assumptions made during design exceed given limits.

Cyclic verification of the model was first undertaken to ensure the model had been built without error, and that results were accurately reflecting expectation from the input data. Once complete, this permitted cyclic experiments to be run (i.e. all constraint parameters turned off) with distributed cycle times considered. As a result the benchmark production rate could be quantified in terms of jobs per hour (JPH) for each area. At this stage it was already possible to identify where cycle time reductions would provide the biggest return - especially important when considering the limited engineering resource and time available. Figure 3 shows the JPH average and distribution for each zone within the Assembly area.
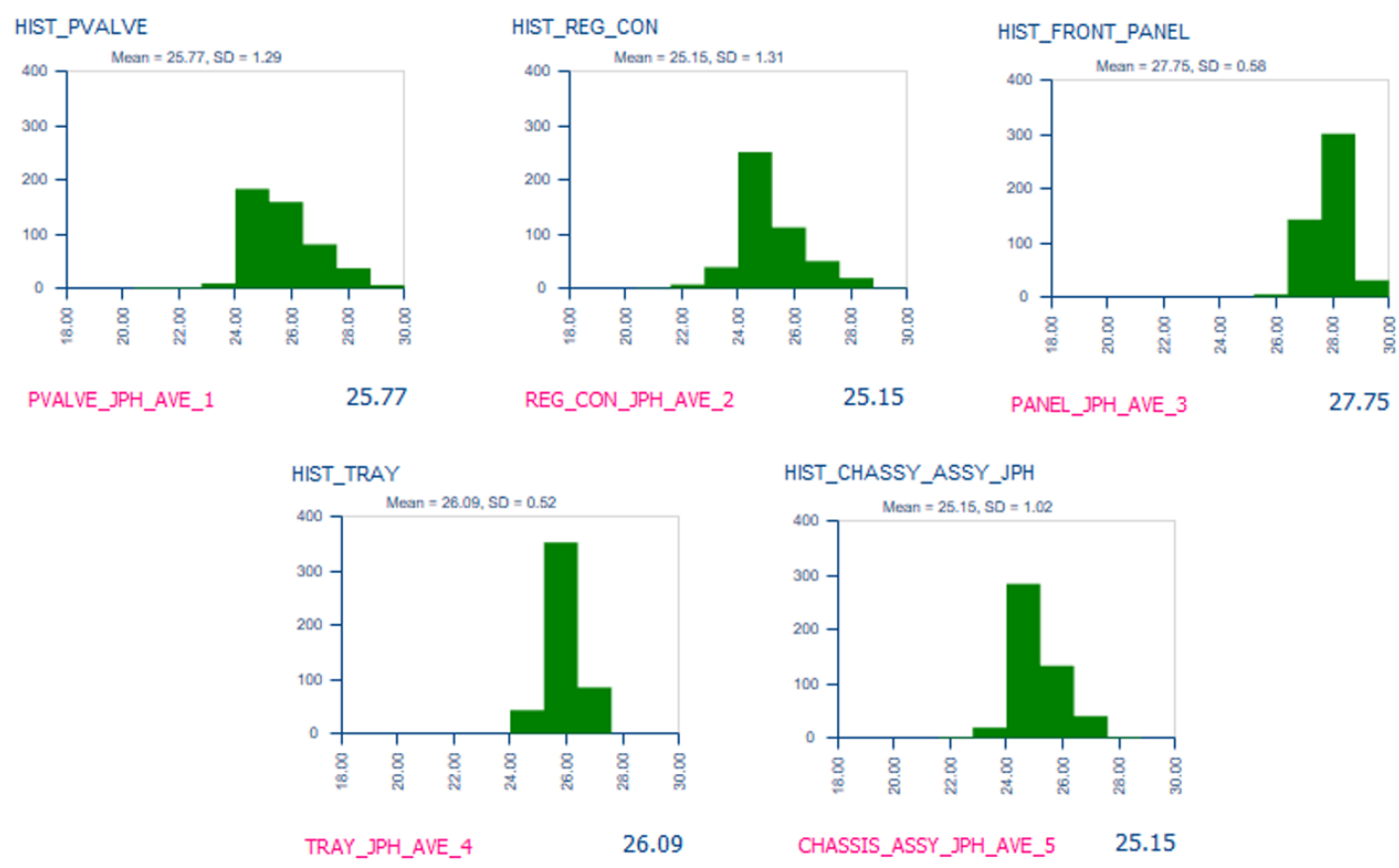

Figure 3. JPH distribution histograms for various areas in the model

Based on these results, information was passed back to the process team demonstrating to them that at a cyclic level the 'regulator and control' zone was constraining the assembly area, and that mitigation would require focus on reducing the cycle time of two stations. Further advice detailing prudent locations for buffers was also provided, in order to keep the constraint areas of the line producing parts.

With the introduction to the model of part rejection rate, direction on how to setup part regression based on initial quality assumptions could be provided. It quickly became clear that rejection rate was going to be a far more significant issue than cycle time, although the data available to support the model would prove to be difficult to secure. Sensitivity analysis on parts rejected per hour and scenario comparison for regression provided clarity on how best to setup the regression strategy. For example, it was shown that given an estimated $10 \%$ rejection rate from a station in the assembly area, the system would not be able to cope should the repair and re-test procedure take place in-station, whereas the introduction of a single repair bay (that included the capability to re-test before being returned to the 
line) would enable the constraint to be overcome. Sensitivity analysis would show that this strategy could cope with up to reject rates as high as $15 \%$ before becoming the system constraint once more.

Similar analysis was undertaken for the test area of the line, while also considering how labour resources should be allocated to the repair areas to maximise efficiency. The compound effect of rejection rates at each station through the process - a total of 17 different work stations each with their own rejection rate across the three areas within test - allowed for prediction of parts per hour into the repair areas to be provided. Sensitivity analysis around the station cycle time, reject rates and repair times could simulate the expected utilisation of labour for repairs and how best to create a labour resource strategy. Initial analysis demonstrated that labour could be shared between repair bays without having a negative impact to overall throughput, so initial estimates could be revised, and labour allocated elsewhere.

\subsection{Commissioning Phase}

After utilising the model throughout planning for estimation of how the line could be expected to perform, and warning of potential areas to closely monitor, it was then used in a slightly different manner once the line had actually started producing ventilators. By shifting away from a nonterminating simulation, experiments could be run to demonstrate what the expected output of the line over a specific period of time would be, and this information was key for understanding requirements for incoming parts as well as when complete ventilators could be shipped to the next stage of the assembly process.

To model projected efficiency improvements over time, dynamic modifiers were applied to both the Erlang cycle time distribution and the station reject rate. By adopting the Monte Carlo concept of simulation (Law and Kelton, 2007) and running multiple replications utilising disparate pseudo-random number streams for sampling, the variation due to randomness in the model could be captured and considered accordingly. This is representative of what would be observed within the facility, as the operators become more familiar with tasks, they are able to complete them with less variability, leading to reduced cycle times and improved quality. The foundation for this analysis was based on the same assumptions made during the planning phase with respect to cycle time and reject rate, and the incremental efficiency improvements aligned to the standard Ford Powertrain processes. It also made it possible to activate additional work stations as they were commissioned and increased the capacity of the line, as well as modelling behaviour between different shifts and shift patterns.

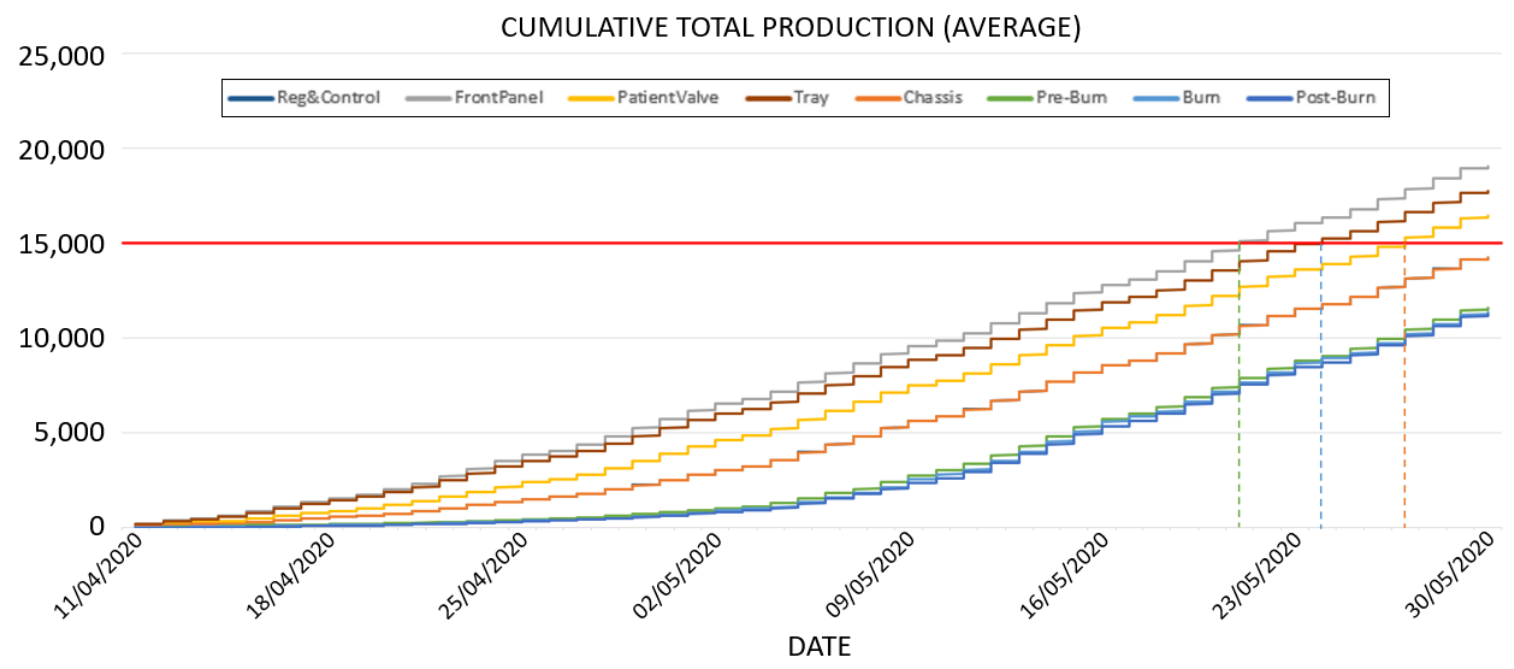

Figure 4. Predicted production over time from each area

An example of the model output can be observed in Figure 4. This chart shows expected output overtime for each of the areas across assembly and test aggregated across 6 replications, showing how production would increase over time and when forecasted totals could expect to be reached within each area. Utilising the simulation in this way was more powerful than trying to calculate throughput 
mathematically as it enabled the variation in the system to be more clearly captured. By running scenarios for multiple replications, the number of parts produced could be predicted within confidence intervals so that plans could be made to cover different scenarios within the range (Figure 5).

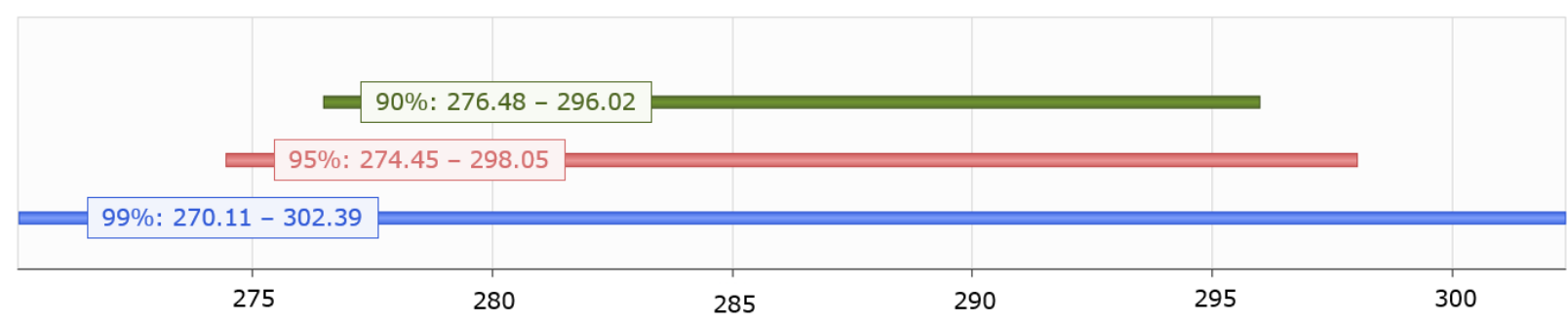

Figure 5. Estimated parts produced, with confidence intervals within a specified time period

\subsection{Production Phase}

As production began to ramp up and the output increased in line with predictions, it became clear that some early model assumptions had not been realised. Although cycle times had improved in line with expectations, external factors (beyond the control of Ford) had resulted in part quality issues in excess of expectation, and a new, more comprehensive regression and repair strategy was required. Again, DES was used to support this development.

Data collection with respect to ventilator repairs provided valuable insight into how units were managed whilst away from the mainline. As this pool of data grew, a fuller understanding of the offline regression process was developed. In conjunction with the test station operator repair guides, which detailed the process, duration, and outcome scenarios of tests conducted in-station, it became possible to develop probability routing distributions for every test station on the line.

When a unit entered a test station it was now possible to determine the probability of whether it would pass all tests, require re-testing, require regression or repair (and where that would be conducted), what the next step in its journey through the line would be (pass to the next station, return to a previous operation, redirect to another area, or take away for offline regression), as well as the time it would take to undertake these steps.

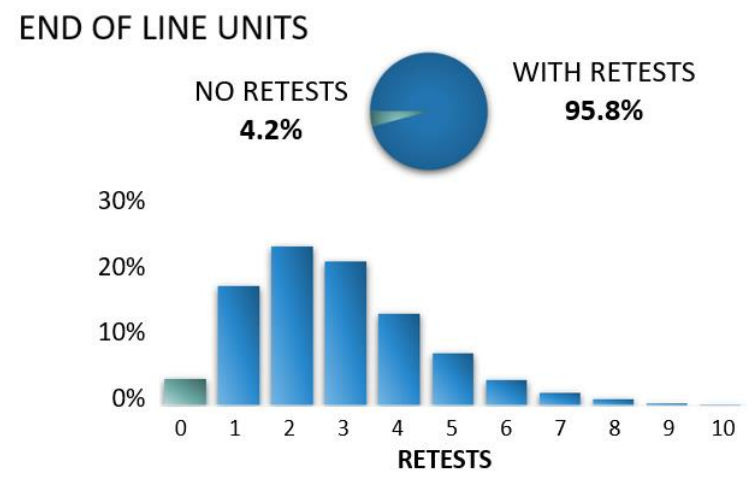

\section{SENT TO HOLDING}
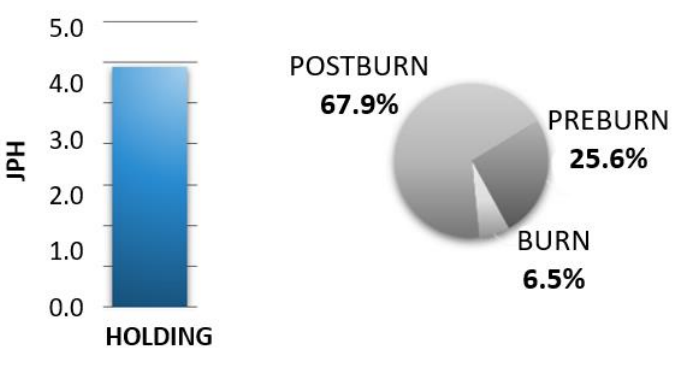

Figure 6. Example screenshot from simulation results dashboard detailing test/repair data

By enhancing the model with these routing probability distributions (which, in line with the continued ethos of rapid response could be modified as soon as new data was available), the impact to production of different strategies could be predicted with confidence. Various solution scenarios put forward by stakeholders across the project could be modelled to see which would result in the biggest improvements to end of line throughput, and the effects viewed in real time during a simulation run from the results dashboard of the Excel 'Front End' (see Figure 6). 


\subsection{Decommissioning Phase}

As the end of the project neared, focus shifted away from production optimisation and onto how the facility could be decommissioned. With the simulation model able to derive dock-to-dock times (also known as total cycle times, the cumulative time taken to complete a process, including wait time and inventory time) at each stage, it could be determined when units/parts had to commence specific processes in order to ensure they were completed in time.

Figure 7 shows a feature of the model whereby a decommission date and date could be entered, and the corresponding date and time that the last parts ought to be loaded to the line in that area, in order to complete production before decommission. This was useful information for the production team as it could support scheduling of parts, allocation of labour and detail exactly when different areas of the line could be switched off.

\begin{tabular}{rcc} 
& \multicolumn{1}{c}{ DATE } & TIME \\
DECOMMISSION: & Thu 2 July & 09:00 \\
AREA & LAST UNIT IN & LAST UNIT OUT \\
\hline PATIENT VALVE & Wed 1 Jul, 17:01 & Wed 1 Jul, 17:11 \\
REGULATOR CONTROLLER & Wed 1 Jul, 11:18 & Wed 1 Jul, 13:42 \\
FRONT PANEL & Wed 1 Jul, 12:38 & Wed 1 Jul, 13:10 \\
TRAY & Wed 1 Jul, 12:45 & Wed 1 Jul, 13:07 \\
CHASSIS & Wed 1 Jul, 15:11 & Wed 1 Jul, 17:16 \\
PRE-BURN & Wed 1 Jul, 17:17 & Wed 1 Jul, 19:20 \\
BURN & Wed 1 Jul, 19:20 & Thu 2 Jul, 05:23 \\
POST-BURN & Thu 2 Jul, 05:23 & Thu 2 Jul, 07:46
\end{tabular}

Figure 7. Example screenshot from simulation results dashboard detailing decommissioning dates/times

\section{CONCLUSION}

Throughout all stages of the project, from conceptualisation to decommissioning, the Ford EOS 2 ventilator facility was supported by Discrete Event Simulation. The developed DES models were able to accurately represent the interference and combination of events that could occur; especially in comparison to static mathematical models. Having a simulation model available through all phases of the project enabled a comprehensive understanding of both current status and future performance, and allowed productivity to be maximised through data-driven decision making.

Notwithstanding, the project did highlight areas where improvements could be made. Due to the nature of the project, the constant rapid evolution of the facility often outpaced the ability to capture and model these changes - despite the inherent flexibility designed into the model to address this need. With non-stop production, and wide changes being implemented on decisions taken by those 'on the ground' on an hour-to-hour basis, at times it was simply not possible to effect the level of responsiveness required from the simulation.

A key aspect of this was data capture. The speed at which the facility was installed did not provision for an adequate data infrastructure to be installed. At the outset, there was no data available meaning substantive assumptions had to be made, and despite the engineering expertise of those making the assumptions, these were not always correct. As data later became available once production increased, it was not always reliable nor accessible in a suitable timeframe. A key takeaway from this project has been the criticality of viable data, available in a timely fashion, in order to support a simulation model's development.

Those issues aside, the project was still considered a success for the PTME Simulation team. It had been demonstrated that a valid DES model could be swiftly built and used to support a low volume, 'pop-up' manufacturing facility. Going forward it gives confidence that for similar projects the team 
has the experience and insight to specify the requirements of such a facility at a conceptual stage, and maintain a strategic position to enable the execution of data-driven engineering decisions.

\section{REFERENCES}

Department for Business, Energy \& Industrial Strategy. (2020). Call for businesses to help make NHS ventilators. https://www.gov.uk/government/news/production-and-supply-of-ventilators-andventilator-components, accessed February 2021.

Ferguson NM, et al. (2020). Impact of non-pharmaceutical interventions (NPIs) to reduce COVID-19 mortality and healthcare demand. Imperial College COVID-19 Response Team. https://doi.org/10.25561/77482

Higgins, M (2013). Fitness of simulation: advancing the application of discrete event simulation within the automotive industry. MPhil Thesis, Cardiff University. http://orca.cf.ac.uk/61450/

Ladbrook, J. and A. Januszczak (2001). Fords Power Train Operations - Changing the Simulation Environment. The Proceedings of the 2001 Winter Simulation Conference Washington, pp 863-869

Law, A. M. and Kelton, W. (2007) Simulation Modelling and Analysis. Boston: McGraw-Hill

Robinson, S. (2007). Simulation the Practice of Model Development and Use. 2007, UK: John Wiley and Sons.

\section{AUTHOR BIOGRAPHIES}

MATTHEW WINDELER graduated with a Master of Science in Materials Science and Engineering in 2014. He has worked with Ford Motor Company since 2014, initially gaining experience within Powertrain Manufacturing Engineering (PTME) as a tooling engineer within Process Engineering, before taking on his current role as Senior Simulation Engineer in 2018. He is the lead engineer responsible for delivery of simulation models to support manufacturing process feasibility and commissioning, as well as engaging with academic partners to further develop application of the Simulation toolsets within Ford. His email address is <mwindele@ford.com>.

MICHAEL HIGGINS graduated with a Bachelor of Science in Mathematics and Physics in 2011 and in 2014 gained an MPhil(Eng) through his research into the application of Discrete Event Simulation within the automotive industry. He has worked with Ford Motor Company since 2012 where his current role is within Powertrain Manufacturing Engineering (PTME) as Simulation and Process Optimisation Leader. He has gained a holistic view of powertrain manufacturing and has utilised simulation as a tool across several functions within PTME including plant and production engineering roles. He is currently researching and developing methods to increase usability, and widen the application of, the Ford Simulation Toolset. His email address is <mhiggi37@ford.com>

GILES THOMAS B.Eng(Hons) is a Manufacturing Engineer and freelance simulation specialist, with over 20 years' experience in DES within the automotive and aerospace industries. Now working with Ford Motor Company, he is responsible for development of their FASTER rapid model building application, as well as leading various simulation projects across the UK and Europe, and delivering training in the use of discrete event simulation tools and techniques. He is currently studying for an MSc in Computer Science with Data Analytics. His email address is <gthom242@ ford.com> 